\title{
Dilute Solution Properties of Styrene-Methyl Methacrylate Copolymers with Different Architecture
}

\author{
Tadao Kotaka, ${ }^{*}$ Takeshi Tanaka, Hiroshi Ohnuma, ${ }^{* *}$ \\ Yoji MuraKami,*** and Hiroshi INAGAKI \\ Institute for Chemical Research, Kyoto University, Uji, Kyoto 611, Japan.
}

(Received December 15, 1969)

\begin{abstract}
The results of an extensive study on solution properties of alternating, statistical and block copolymers of styrene and methyl methacrylate with nearly equimolar composition are described, by placing emphasis upon their dependence on the sequential arrangement of monomers. The $\theta$ temperatures are determined in cyclohexanol by a phaseequilibrium method. Measurements of intrinsic viscosity are made in six different solvents, which are chosen to differ in their behavior toward the parent homopolymers. All data are examined on the basis of current two parameter theories.

The short-range interactions, as given by the characteristic ratios, are expressed for the block copolymers as a composition average of those of the parent homopolymers, whereas for the alternating and statistical copolymers they show a positive deviation from the average. The deviation is roughly proportional to the population of the dyads of unlike monomers in the copolymer chain, i.e., to the run number. The long-range interactions are interpreted by assuming dyads as copolymer segments. Thus, the interaction parameters of the statistical and block copolymers may be expressed as a function of composition and run number in terms of the three parameters each characterizing the interaction of the parent homopolymers and the alternating copolymer with the pure solvent.

KEY WORDS Copolymer / Solution Properties / Styrene / Methyl Methacrylate / Alternating Copolymer / Statistical Copolymer / Block Copolymer / Sequential Arrangement / Short-Range Interaction / Long-Range Interaction /
\end{abstract}

The solution characteristics of copolymers have certain features resulting from the interactions (of both short-range ${ }^{1}$ and long-range ${ }^{2,3}$ nature) between chemically different monomers in the copolymer chains. The overall effects of such interactions should be dependent not only on the composition but also on the sequential arrangement of monomers along the chain. ${ }^{4}$ A number of studies ${ }^{4-10}$ on binary copolymers have given a description of their interaction parameters as a function of molecular weight and composition

* To whom correspondence should be addressed.

** Present address: Department of Physics, Faculty of Science, Nagoya University, Nagoya, Japan.

*** Present address: Mihara Mill, Teijin and Co., Mihara, Hiroshima, Japan. in terms of those characteristic of their parent homopolymers. Relatively few studies are concerned specifically with their dependence on the sequential arrangement of monomers, although recent experiments ${ }^{11-13}$ have suggested that the dependence is of prime importance.

The difficulty in carrying out a quantitative study on this problem is presumably due in part to the difficulty in preparing, from a given monomer pair, different model copolymers, each of which has a given composition but a definitely different arrangement of the monomers. Recent development in the technique of copolymer synthesis, ${ }^{14}$ however, has opened a wide possibility: For example, in addition to statistical and block copolymers of styrene (St) and methyl methacrylate 
(MMA), both of which may be prepared by conventional methods, ${ }^{15,16}$ a new technique developed by Hirooka ${ }^{14}$ allows one to prepare a model copolymer of strictly alternate sequence - $-\mathrm{St}$ MMA-St-MMA-, i.e., the alternating copolymer.

We have prepared three different model copolymers of St and MMA with nearly equimolar composition, and have studied their solution properties, placing emphasis on examining their dependence on the sequential arrangement of the monomers. We will report the results herein. The conclusions drawn would be applicable to any amorphous (noncrystallizable) linear copolymers that are composed of a monomer pair, the parent homopolymers of which are to some extent incompatible with each other.

\section{EXPERIMENTAL}

Preparation and Fractionation of Model Copolymers

Block copolymers consist of linear chains of poly (methyl methacrylate) - polystyrene - poly (methyl methacrylate) type (coded as series B) prepared by an anionic polymerization technique. ${ }^{16}$ A bifunctional initiator, sodium biphenyl, and tetrahydrofuran were used. A precaution was taken to avoid grafting of polystyrene (PSt) on poly (methyl methacrylate) (PMMA) blocks. ${ }^{17}$ The details are described elsewhere. ${ }^{13 a, b}$ Whenever necessary, we used PSt and PMMA homopolymers (coded as series $\mathrm{H}$ and $\mathrm{M}$, respectively) prepared by the same anionic polymerization technique.

Statistical copolymers with nearly azeotropic composition (St-mole fraction $m=0.52 \pm 0.01$ ) are fractionated samples (coded as series SMA) from low conversion products prepared by a radical copolymerization method, which was described previously. ${ }^{8}$ Our stock samples ${ }^{8}$ coded as series SM3 $(m=0.29 \pm 0.01)$, SM5 $(m=0.56 \pm 0.01)$, and SM7 ( $m=0.70 \pm 0.01)$ were used, whenever necessary for the sake of comparison.

Alternating copolymers were prepared by a method reported by Hirooka. ${ }^{14}$ First, MMA monomer was allowed to form complex with ethyl aluminum sesquichloride* in toluene at

\footnotetext{
* By the courtesy of Dr. Hirooka, Central Research Laboratories, Sumitomo Chemical Co., Ltd., Takatsuki, Osaka.
}

$-78^{\circ} \mathrm{C}$. The temperature was adjusted to a desired value ( $c f$. Table I); then St monomer was introduced into the complex mixture. Occasionally a small amount of benzoyl peroxide (BPO) was added as a cocatalyst for controlling molecular weight of the product. The mixture reacted spontaneously, yielding a high molecular weight product. The reaction was terminated by pouring the mixture into an excess of methanol. The product was recovered and purified by twofold precipitation from a butanone solution into methanol-water (1:1 by volume) mixture that was acidified with a few drops of hydrochloric acid. The precipitate was washed thoroughly with the acidified methanol-water mixture and dried in vacuum at about $50^{\circ} \mathrm{C}$. Table I summarizes the copolymerization data.

Each whole copolymer was subjected to successive fractionating precipitation by using butanone and methanol as a solvent and nonsolvent, respectively. The condition was adjusted so that, for example, the initial precipitation took place at $30^{\circ} \mathrm{C}$ from $0.48 \%$ solution with the volume fraction of methanol being 0.255 . In each case, five or seven fractions were isolated, each being purified by the same procedures as for the whole copolymer. Even after the fractionation, an initial few fractions appeared slightly turbid as redissolved in butanone, presumably because of a last trace of catalyst fragments. Each of such turbid solutions was centrifuged with a Hitachi Model 55P preparatory centrifuge at about $16000 \mathrm{rpm}$ for 90 minutes. Then the fraction in clear supernatant was recovered. The fractions were coded as series AL.

As a test for the microstructure of the copolymer samples, NMR spectra were taken by a $100 \mathrm{mc} / \mathrm{s}$ high-resolution NMR spectrometer Model JNM4H-100 (Japan Electron Optics Laboratories Inc.) at about $80^{\circ} \mathrm{C}$ on about $10 \%$ solution in deuterated chloroform with tetramethyl silane as an internal standard. The results are shown in Figure 1.

\section{Measurements}

The composition (St-mole fraction $m$ or weightfraction $x$ ) of each fraction was determined from carbon content by repeating combustion analysis several times. Osmotic-pressure measurements were made by a Mechrolab Model 502 high speed 
St-MMA Copolymers with Different Architecture

Table I. Polymerization data of St-MMA alternating copolymers

\begin{tabular}{|c|c|c|c|c|c|c|c|c|c|c|}
\hline \multirow[b]{2}{*}{ Code } & \multicolumn{2}{|c|}{ Monomer (g) } & \multicolumn{2}{|c|}{ Catalyst (g) } & \multirow{2}{*}{$\begin{array}{c}\begin{array}{c}\text { Solvent } \\
(\mathrm{m} l)\end{array} \\
\text { [Toluene] }\end{array}$} & \multirow{2}{*}{$\begin{array}{c}\text { Temp. } \\
\left({ }^{\circ} \mathrm{C}\right)\end{array}$} & \multirow{2}{*}{$\begin{array}{l}\text { Time } \\
\text { (min) }\end{array}$} & \multirow{2}{*}{$\begin{array}{l}\text { Yield } \\
(\mathrm{g})\end{array}$} & \multicolumn{2}{|c|}{ Product } \\
\hline & St & MMA & $\mathrm{AlEt}_{1.5} \mathrm{Cl}_{1.5}$ & BPO & & & & & $\begin{array}{l}\text { St-content } \\
\text { (mole frac- } \\
\text { tion) }\end{array}$ & $\begin{array}{l}{[\eta]^{\mathrm{a}}} \\
(\mathrm{d} l / \mathrm{g})\end{array}$ \\
\hline ALI & 75.5 & 48.4 & 30 & 0.400 & 150 & 30 & 60 & 75.6 & 0.495 & 0.957 \\
\hline ALII & 127 & 47 & 30 & 1.50 & 150 & 20 & 40 & 40.0 & 0.505 & 0.816 \\
\hline ALIII & 71 & 48.4 & 23 & 0 & 53 & -78 & 900 & 10.0 & 0.480 & 3.00 \\
\hline
\end{tabular}

a Measured in toluene, at $30.0^{\circ} \mathrm{C}$.

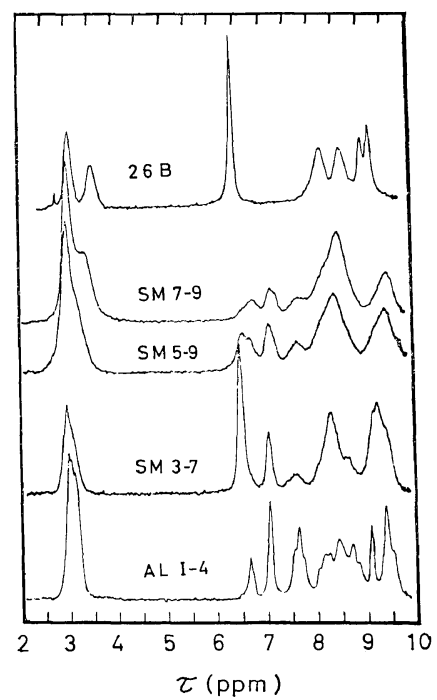

Figure 1. High-resolution NMR spectra of St-MMA model copolymers as measured in deuterated chloroform solution at $80^{\circ} \mathrm{C}$. See text for sample codes.

membrane osmometer. Membranes used were properly conditioned Ultracellafilter grade feinst or allerfeinst. For low molecular-weight solutes, a Bac-T-Flex B20 membrane filter was used. Refractive-index increments were measured for $436 \mathrm{~m} \mu$ light with a Shimadzu differential refractometer of the Debye type. Light-scattering measurements were made by a Shimadzu lightscattering photometer of the modified Brice type. Intrinsic viscosity measurements were made by Ubbelohde dilution viscometers. All these measurements were carried out according to our laboratory routine, which is described elsewhere in greater detail. ${ }^{8,13}$

Previously the determination of $\theta$ temperatures of St-MMA copolymers was made by osmometry as the temperature at which the second virial coefficient $A_{2}$ vanishes. ${ }^{28,8,13 \mathrm{c}}$ For the three model copolymer- and the parent homopolymer-cyclohexanol (CHL) systems, the determination was made by measurements of critical-miscibility temperature versus molecular weight relationships. In each measurement, about $3 \mathrm{ml}$ of solution was sealed in a glass tube. The tube was immersed in a bath, first kept at about $90^{\circ} \mathrm{C}$. The temperature was gradually lowered (at a rate of about $0.2^{\circ} \mathrm{C} / \mathrm{min}$ ), while the solution was being constantly shaken. The temperature at which an incipient cloudiness was recognized by visual inspection was recorded as the precipitation temperature. The disappearance of cloudiness upon rewarming was also checked as the dissolution temperature. These two temperatures for a given solution always agreed with each other within 0.1 to $0.3^{\circ} \mathrm{C}$. The results were analyzed by the Flory-Shultz equations. ${ }^{2,19}$

\section{RESULTS}

\section{Characterization of Model Copolymers \\ Fractionation*}

For the series SMA and AL, the composition of each fraction agreed fairly well with the value expected from the copolymerization kinetics; the composition fluctuation was negligible.

For the series SMA, measurements of both osmotic pressure $\pi$ and intrinsic viscosity [ $\eta]$ were made in toluene at $30.0^{\circ} \mathrm{C}$. The double logarithmic plots of number-average molecular weight $M_{n}$ versus [ $\eta$ ] and $A_{2}$ are expressed, respectively, as

$$
\begin{aligned}
& {[\eta]=1.82 \times 10^{-4} M_{n}^{0.69}(\text { SMA-TOL 30.0) }} \\
& A_{2}=2.00 \times 10^{-2} M_{n}{ }^{-0.31}(\text { SMA-TOL 30.0) }
\end{aligned}
$$

The osmometric dimensionless ratios are approximated as $A_{2} M_{n} /[\eta]=110$, which is in agreement with typical values for homopolymer solu-

\footnotetext{
* Numerical data are available upon request.
} 
tions. For the series $\mathrm{AL}$, measurements of $\pi$ were made in 1-chloro- $n$-butane at $30.0^{\circ} \mathrm{C}$, and of $[\eta]$ in toluene at $30.0^{\circ} \mathrm{C}$, which is expressed as

$$
[\eta]=0.933 \times 10^{-4} M_{n}^{0.76}
$$

These results show that the fractionation procedures for both series were satisfactory for separating them according to molecular weight. From each series several fractions were selected for later experiments. The average composition was $m=0.52 \pm 0.01$ for seven fractions selected from the series SMA, and $m=0.50 \pm 0.01$ for six fractions from the series AL.

Each sample of the series B was prepared by a batch-wise synthesis, and was not subjected to fractionation. However, each sample was purified to remove free PSt and PMMA by solvent extraction technique. ${ }^{13 a, b}$ The composition of each sample ranges from $m=0.45$ and 0.53 for five samples, and two other samples with $m=0.41$ and 0.61 were also used. The characterization data are described elsewhere. ${ }^{13 a, b}$

Weight-Average Molecular Weight, Compositional and Molecular-Weight Heterogeneity*

For a copolymer with compositional heterogeneity, the light-scattering molecular weight $M_{a p p}$ as obtained by applying the Zimm procedure ${ }^{20}$ is not a weight-average but an apparent value which depends on the solvent used for the measurement. $^{4,5,21}$ Table II summarizes light- scattering data on some samples. As anticipated from the mechanism of the alternating copolymerization, ${ }^{14}$ fractions from the series $\mathrm{AL}$ should be homogeneous in composition. In such a case the values of $M_{a p p}$ should be weight-average $M_{w}$ and independent of solvent. The effect of compositional heterogeneity on $M_{a p p}$ is negligible also in low-conversion statistical copolymers, ${ }^{4,22}$ and especially in a favorable solvent such as butanone for St-MMA copolymers. Therefore, for the series SMA the values of $M_{a p p}$ in butanone are used as $M_{w}$. The results for the Series SMA and $\mathrm{AL}$ show that the compositional heterogeneity is negligible for both cases, and the polydispersity in molecular weight is moderate as judged from the values of $M_{w} / M_{n}$ ( $c f$. Table IV).

As opposed to them, the compositional and molecular-weight heterogeneities of block copoly-

\footnotetext{
* See footnote on page 247.
}

mer samples must be closely correlated with each other. The analysis of the $M_{a p p}$ and other data, which was discussed previously, ${ }^{13 a, b}$ shows that the heterogeneity of each sample is reasonably small except for a few cases. The values of $M_{w} / M_{n}$ are 1.2 to 1.6 for the six samples used for $[\eta]$-measurements ( $c f$. Table IV).

\section{Sequential Arrangement of Monomers}

The sequential arrangement of monomers in a copolymer chain may be conveniently described in terms of Harwood-Ritchey's run number, ${ }^{23}$ which is equal to the probability of finding dyads of unlike monomers in the copolymer chain. At the moment no physical method is available for a direct determination of the probability. However, it may be surmised from the knowledge of monomer reactivity ratios on the basis of an appropriate scheme for copolymerization kinetics. $^{15,23}$ For example, employing the so-called terminal model which involves only two reactivity ratios $r_{a}$ and $r_{b}$, one obtains the run number $2 r=P_{a b}+P_{b a}\left(P_{a b}=P_{b a}\right)$ as

$$
2 r=\frac{4 m(1-m)}{1+\left\{1+4 m(1-m)\left(r_{a} r_{b}-1\right)\right\}^{1 / 2}}
$$

where $m$ is the mole fraction of monomer $a$ (in this case $\mathrm{St}$ ) in the product copolymer, and $\boldsymbol{P}_{a b}$ is the probability of finding $a-b$ dyads. The Eq. 1 is readily obtained by eliminating the feedcomposition from the feed-product compositional relationship ${ }^{15,23}$ and the Harwood-Ritchey equation for the feed-composition versus run number relationship. ${ }^{23}$

The series AL should have completely alternate sequences of St and MMA, i.e., $m=0.5$ and $2 r=1.00$, as Hirooka ${ }^{14}$ has pointed out for the following reasons: (i) Neither the MMA-catalyst complex nor the St-monomer alone does polymerize, and the polymerization proceeds only when these two species are mixed; thus $r_{a}=r_{b}=0$. (ii) The product-composition is always found to be $m=0.5$, regardless of the feed-composition (cf. Table I). (iii) Infrared absorption spectra are practically identical to an equimolar statistical copolymer. The identity implies that unusual bonds or atomic groups are absent in the copolymer chain. (iv) On the other hand, high resolution NMR spectra are entirely different, as demonstrated in Figure 1. Among several features of 
interest, two of them are especially noteworthy. The diamagnetic shift (in 3.0-3.5 $\tau$ region) of the ortho phenyl-proton reasonance is not observed in the spectrum of the alternating copolymer, as opposed to those of the other copolymers. The spectrum of the alternating copolymer is lacking a methoxy peak at $6.4 \tau$ which has been assigned to MMA triads in those of block copolymer and statistical copolymers with low St-content. ${ }^{14,24}$ These facts imply that the alternating copolymer chain, as it should, has no St- and MMA-triads, which are always found in the corresponding statistical and block copolymer chains. ${ }^{14}$

A large accumulation of evidence from studies on copolymerization kinetics, ${ }^{15}$ on high-resolution NMR spectroscopy, ${ }^{24}$ and especially recent evidence from thin layer chromatography ${ }^{25}$ suggests that most conventional radical copolymerizations are satisfactorily described by the terminal model. Thus we employed reported values ${ }^{15}$ $r_{a}=0.52$ and $r_{b}=0.46$ for the radical copolymerization of St and MMA, and computed the run number by Eq. 1. The results are listed in Table III. For block copolymers the run number may be regarded as practically zero.

\section{Thermodynamic and Conformational Properties $\theta$ Temperatures*}

The critical-miscibility temperature $T_{c}$ for each sample in CHL was determined as the maximum of precipitation temperature versus polymer concentration $v_{p}$ (by volume fraction) curve. Values of $v_{p}$ were calculated from the specific volumes of the polymer and solvent by neglecting volume change upon mixing. Since the specific volumes of St-MMA copolymers in CHL were unavailable, we calculated them from the values of $\mathrm{PSt}^{26 \mathrm{a}}$ and PMMA $^{26 \mathrm{~b}}$ with a further assumption of additivity in the volumes with respect to composition. The $T_{c}$ versus $M_{n}$ data are plotted, as shown in Figure 2, according to the Flory-Shultz equation, 2,19

$$
\frac{1}{T_{c}}=\frac{1}{\theta}\left(1+b M_{n}^{-1 / 2}\right) ; \quad b=\frac{\left(V_{s} / \bar{v}\right)^{1 / 2}}{\psi}
$$

where $V_{s}$ is the molar volume of the solvent; $\bar{v}$ is the partial specific volume of the polymer; and $\phi$ is the entropy of the mixing papameter. From these values, the enthalpy parameter $\kappa$ and the

\footnotetext{
* See footnote on page 247.
}

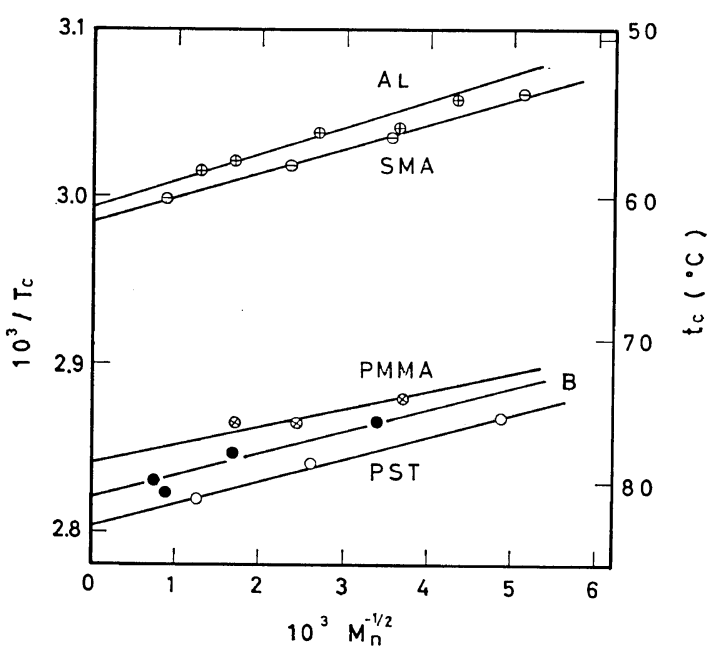

Figure 2. Plots of the reciprocal of the critical temperature $T_{c}^{-1}$ vs. $M_{n}^{-1 / 2}$ according to Eq. 2 for St-MMA model copolymers with nearly equimolar composition and the parent homopolymers in cyclohexanol. See text for sample codes.

free energy parameter $\chi$ are computed as $\kappa=\psi \theta / T$ and $\chi=1 / 2-(\phi-\kappa)$.

In view of our previous studies ${ }^{8,13,18}$ the concept of the $\theta$ condition for homopolymer systems is valid at least for alternating and statistical copolymers: The Flory-Shultz equation should also be applicable. However, there is a doubt as to whether this is also true for block copolymers. Thus we simply regard Eq. 2 as an operational definition of estimating the interaction parameters. The results are summarized in Table III together with previous osmometric data. ${ }^{18}$ For all the systems, the values of $\theta$ obtained from the two experiments are in satisfactory agreement with each other, while the values of $\phi$ from $T_{c} v s . M$ data are much larger than those from $A_{2} v s . T$ data, as usually found in homopolymer solutions. ${ }^{2}$

It is interesting to note that the $\theta$ temperatures of PSt and PMMA in this solvent, CHL, are quite close with each other, ${ }^{7,18}$ and those of the block copolymers are also quite close to them, showing negligible dependence on composition. ${ }^{13 c, 18}$ The fact suggests that all the intra- and interchain interactions in the block copolymer would possibly vanish simulteneously at this temperature: A real $\theta$ state might be realized. It should be noted, however, that this was rather a fortuitous 
event, and is not necessarily true in other (pseudo) $\theta$-solvents such as ethoxyethanol. ${ }^{13 c, 18}$ On the other hand, the $\theta$ temperatures of St-MMA statistical copolymers are dependent on composition, and they are far lower than either of those of the parent homopolymers. ${ }^{7,18}$ The $\theta$ temperature of the series AL is slightly but distinctly lower than that of the series SMA, in spite of the similarity in their composition. ${ }^{12}$ Apparently this is due to the difference in the se-

Table II. Light-scattering data on some model copolymer samples ${ }^{\mathrm{a}}$

\begin{tabular}{|c|c|c|c|c|c|c|c|c|c|}
\hline Code & $\begin{array}{c}\text { St-content } \\
m\end{array}$ & $10^{-4} M_{n}$ & $10^{-4} M_{w}$ & Solvent ${ }^{b}$ & $10^{-4} M_{a p p}$ & $10^{4} A_{2}^{\prime} a p p$ & $10^{8}\left\langle s^{2}\right\rangle_{a p p}^{1 / 2}$ & {$[\eta]$} & $10^{-21} \Phi$ \\
\hline \multicolumn{10}{|c|}{ Alternating copolymers (AL) } \\
\hline III-2 & 0.492 & - & 233 & MEK & 233 & 0.980 & 603 & 2.63 & 2.60 \\
\hline \multirow{2}{*}{ III-3 } & 0.490 & 137 & 142 & MEK & 142 & 1.02 & 470 & 1.905 & 2.43 \\
\hline & & & & TOL & 150 & 1.27 & 575 & 3.20 & 2.37 \\
\hline \multirow[t]{2}{*}{ I-1 } & 0.490 & 57.2 & 80.0 & MEK & 80.0 & 1.26 & 367 & 1.310 & 2.10 \\
\hline & & & & TOL & 83.0 & - & - & - & - \\
\hline \multicolumn{10}{|c|}{ Statistical copolymers (SMA) } \\
\hline \multirow[t]{2}{*}{$6-22$} & 0.513 & 135 & 180 & MEK & 180 & 1.45 & 512 & 2.34 & 3.01 \\
\hline & & & & TOL & 180 & 1.63 & 665 & 3.61 & 3.20 \\
\hline \multirow[t]{2}{*}{$6-4$} & 0.517 & 76.9 & 83.5 & MEK & 83.5 & 1.70 & 358 & 1.432 & 1.99 \\
\hline & & & & TOL & 89.0 & 1.66 & 392 & 2.00 & 2.48 \\
\hline $7-3$ & 0.515 & 50.2 & 61.7 & MEK & 61.7 & 1.66 & 287 & 1.083 & 2.44 \\
\hline \multirow[t]{2}{*}{$2-2$} & 0.512 & 35.8 & 47.0 & MEK & 47.0 & 2.43 & 293 & 0.914 & 1.61 \\
\hline & & & & TOL & 52.3 & 2.15 & 320 & 1.274 & 1.71 \\
\hline \multicolumn{10}{|c|}{ Block copolymers (B) } \\
\hline \multirow[t]{2}{*}{ 27B } & 0.404 & 124 & 147.4 & MEK & 145 & 1.07 & 456 & 1.925 & 2.76 \\
\hline & & & & TOL & 141 & 1.01 & 402 & 2.595 & - \\
\hline \multirow[t]{3}{*}{ 22B } & 0.442 & 58.2 & 80.7 & MEK & 76.4 & 1.39 & 352 & 1.28 & 2.36 \\
\hline & & & & DCE & 76.9 & - & 366 & - & - \\
\hline & & & & TOL & 82.6 & 0.89 & 278 & 1.73 & - \\
\hline \multirow[t]{3}{*}{ 26B } & 0.450 & 25.9 & 30.8 & MEK & 30.6 & 1.48 & 224 & 0.635 & 1.47 \\
\hline & & & & DCE & 33.4 & 2.54 & 248 & - & 一 \\
\hline & & & & TOL & 32.8 & 1.70 & 181 & 0.827 & - \\
\hline \multirow[t]{3}{*}{$25 B$} & 0.481 & 13.5 & 26.0 & MEK & 25.0 & 1.74 & 208 & 0.445 & 1.56 \\
\hline & & & & DCE & 25.6 & 2.27 & 302 & - & - \\
\hline & & & & TOL & 28.2 & 1.14 & 195 & 0.633 & - \\
\hline
\end{tabular}

a All measurements were made at $30.0^{\circ} \mathrm{C}$.

b MEK: Butanone; TOL: Toluene; DCE: Dichloroethane.

Table III. Thermodynamic data for St-MMA model copolymer-cyclohexanol systems

\begin{tabular}{|c|c|c|c|c|c|c|c|c|c|c|}
\hline \multirow{2}{*}{ Code } & \multirow{2}{*}{$\begin{array}{c}\text { Number of } \\
\text { samples } \\
\text { (Range of } 10^{-4} M_{n} \text { ) }\end{array}$} & \multirow{2}{*}{$\begin{array}{l}\text { St-Content } \\
m\end{array}$} & \multirow{2}{*}{ 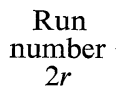 } & \multicolumn{4}{|c|}{$T_{c}$ vs. $M_{n}$ data } & \multicolumn{3}{|c|}{$A_{2}$ vs. $T$ data $^{\mathrm{a}}$} \\
\hline & & & & $\Theta\left({ }^{\circ} \mathrm{C}\right)$ & $\psi$ & $\kappa\left(81^{\circ} \mathrm{C}\right)$ & $\chi\left(81^{\circ} \mathrm{C}\right)$ & $\Theta\left({ }^{\circ} \mathrm{C}\right)$ & $\phi$ & $\chi\left(81^{\circ} \mathrm{C}\right)$ \\
\hline PSt & $3(4.20-54.8)$ & 1.00 & 0.00 & 83.0 & 2.26 & 2.27 & 0.51 & 81.8 & 0.73 & 0.505 \\
\hline PMMA & A $3(7.21-34.3)$ & 0.00 & 0.00 & 78.8 & 3.62 & 3.60 & 0.48 & 79.4 & 1.39 & 0.499 \\
\hline $\mathrm{AL}$ & $5(6.05-57.2)$ & $0.50 \pm 0.01$ & 1.00 & 60.8 & 2.04 & 1.92 & 0.38 & - & - & - \\
\hline SMA & $4(3.85-135)$ & $0.52 \pm 0.01$ & 0.67 & 61.8 & 2.19 & 2.08 & 0.39 & 61.3 & 0.56 & 0.468 \\
\hline B & $4(8.66-117)$ & $0.50 \pm 0.10$ & 0.00 & 80.8 & 2.42 & 2.42 & 0.50 & 81.3 & 0.87 & 0.505 \\
\hline SM3 & $1(35.4)$ & 0.285 & 0.51 & - & - & - & - & 68.2 & 1.10 & 0.495 \\
\hline SM5 & $1(35.0)$ & 0.552 & 0.66 & - & 一 & - & - & 61.3 & 0.57 & 0.470 \\
\hline SM7 & $1(34.2)$ & 0.694 & 0.52 & 一 & - & - & - & 63.0 & 0.56 & 0.473 \\
\hline
\end{tabular}

a See refs. 8, 13c, and 18: Froelich and Benoit reported that $\Theta=83.5$ for PSt and 77.6 for PMMA (ref. 7). 
quential arrangement between these two copolymers.

\section{Light-Scattering Data}

Table II summarizes light-scattering data on some samples obtained at $30.0^{\circ} \mathrm{C}$. Generally the Zimm procedure ${ }^{20}$ applied to copolymer data does not provide a mean-square statistical radius $\left\langle s^{2}\right\rangle$ but only an apparent radius $\left\langle s^{2}\right\rangle_{a p p .} .^{21 b, c}$ However, $\left\langle s^{2}\right\rangle_{a p p}$ for alterating and statistical copolymers need not be distinguished from $\left\langle s^{2}\right\rangle$, because in both cases constituent monomers are distributed uniformly along the copolymer chain. Thus the apparent radius $\left\langle s^{2}\right\rangle_{a p p}$ should be corrected only against polydispersity in molecular weight by the formula suggested by $\mathrm{Zimm}^{20}$ to estimate the weight-average value $\left\langle s^{2}\right\rangle_{w}$. From these data the Flory-Fox viscosity constant ${ }^{2} \Phi$ was computed as

$$
\Phi=\frac{[\eta] M_{w}}{\left[6\left\langle s^{2}\right\rangle_{w}\right]^{3 / 2}}
$$

The values are estimated for the series $\mathrm{AL}$ and SMA as about $10^{-21} \Phi=2.0-2.8$ ( $c f$. Table II), which are roughly in agreement with those usually found for homopolymers. ${ }^{2}$ As discussed previously, ${ }^{13 b}$ the situation for the block copolymers is somewhat different. The values of $\Phi$ in butanone (MEK) are about the same as for the series $\mathrm{AL}$ and SMA, whereas those in toluene (TOL) are considerably larger than the usual values. The latter result may not be surprising, because PMMA are practically invisible in TOL (i.e., the specific refractive-index increment of PMMA in TOL is nearly zero), and $\left\langle s^{2}\right\rangle_{a p p}$ reflects the dimension of the PSt-part alone. The implication of the above results is that even block copolymer chains may assume random coil conformations similar to homopolymer chains at least in such solvents which have similar solubility toward both parent homopolymers. In other words, the incompatible interactions between PSt and PMMA are not strong enough to produce anomalous conformations in such solvent. ${ }^{13}$ In other solvents which have quite different solubility toward each parent homopolymer, block copolymers would assume peculiar conformations or even form aggregates. ${ }^{13}$

\section{Intrinsic Viscosity Data*}

\footnotetext{
* See footnote on page 247.
}

Viscosity measurements were made in six different solvents including a $\theta$ solvent, CHL. The solvents were chosen to differ in their behavior toward the parent homopolymers: Tetrahydrofuran (THF) is a good solvent for both; TOL is a good solvent for PSt and a moderate solvent for PMMA, while MEK is the opposite; 1-chloron-butane (1-CB) is a moderate solvent for PSt and a $\theta$ solvent for PMMA $\left(35.5^{\circ} \mathrm{C}\right),{ }^{27 a}$ while diethylmalonate (DEM) is the opposite $\left(\theta=35.9^{\circ} \mathrm{C}\right.$ for PSt). ${ }^{27 b}$ All the data are summarized in the form of the Mark-Houwink-Sakurada equation, $[\eta]=K M_{w}{ }^{a}$, as listed in Table IV.

\section{Estimation of Interaction Parameters \\ Short-Range Interactions}

Here we estimated interaction parameters from [ $\eta$ ] data, ${ }^{28}$ using viscosity equations proposed for the analysis of homopolymer data. ${ }^{29-34}$ For the short-range interactions, we employed the characteristic ratio defined as

$$
C_{\infty}=\left(\frac{\left\langle r_{0}{ }^{2}\right\rangle}{M}\right)\left(\frac{M_{0}}{2 l^{2}}\right)
$$

where $\left\langle r^{2}\right\rangle_{0}$ is the unperturbed mean-square endto-end distance; $M_{0}$ is the mean molecular weight of monomers; and $l$ is the length of the skeletal bond. To estimate $C_{\infty}$, we used a viscosity equation:

$$
[\eta]=K_{0} M_{w}{ }^{1 / 2} ; \quad K_{0}=\Phi_{0}\left[6\left\langle s^{2}\right\rangle_{0} M_{w}\right]^{3 / 2}
$$

where $\Phi_{0}$ is the viscosity constant under the $\theta$ condition $^{1,2}\left(10^{-21} \Phi_{0}=2.68\right)^{35}$; and $\left\langle s^{2}\right\rangle_{0}$ is the unperturbed mean-square molecular radius, which is related to the end-to-end distance as $\left\langle r^{2}\right\rangle_{0}=$ $6\left\langle s^{2}\right\rangle_{0}$. However, when the relation is applied to copolymers, it should be valid only for those copolymers in which different monomers are uniformly distributed along the chain, i.e., for statistical and alternating copolymers. ${ }^{36}$ For a block copolymer of symmetric BAB type, the two dimensions should be related as

$$
\begin{gathered}
\left\langle r^{2}\right\rangle_{0}=6\left\langle s^{2}\right\rangle_{0}\left\{1+\left(\frac{x}{2}\right)(1-x)(1+x) \delta\left\langle s^{2}\right\rangle_{0}\right\}^{-1} \\
\delta\left\langle s^{2}\right\rangle_{0}=\frac{\left(\frac{\left\langle s^{2}\right\rangle_{0}}{M}\right)_{A}-\left(\frac{\left\langle s^{2}\right\rangle_{0}}{M}\right)_{B}}{x\left(\frac{\left\langle s^{2}\right\rangle_{0}}{M}\right)_{A}+(1-x)\left(\frac{\left\langle s^{2}\right\rangle_{0}}{M}\right)_{B}}
\end{gathered}
$$


where the subscripts $A$ and $B$ denote the quantities characteristic of the parent homopolymers; $x$ is the composition by weight fraction. ${ }^{37}$

It has been often argued that the unperturbed

Table IV. List of Mark-Houwink-Sakurada constants, $[\eta]=K M_{w}{ }^{a}$, of St-MMA model copolymers in various solvents

\begin{tabular}{|c|c|c|c|c|}
\hline Code & $\begin{array}{c}\text { Number of } \\
\text { samples: St- } \\
\text { content } m \\
\text { (Range of } \\
10^{-4} M_{w} \text { and } \\
\left.M_{w} / M_{n}\right) \\
\end{array}$ & Solvent ${ }^{\mathrm{a}}$ & $10^{4} K$ & $a$ \\
\hline \multirow[t]{6}{*}{$\mathrm{AL}$} & $6: 0.50 \pm 0.01$ & THF(25.05) & 0.775 & 0.76 \\
\hline & $(7.06-233)$ & TOL & 1.09 & 0.73 \\
\hline & $(1.29-1.33)$ & MEK & 1.15 & 0.69 \\
\hline & & DEM & 1.50 & 0.66 \\
\hline & & $1-\mathrm{CB}$ & 1.08 & 0.70 \\
\hline & & CHL(61.0) & 8.00 & 0.50 \\
\hline \multirow[t]{5}{*}{ SMA } & $7: 0.52 \pm 0.01$ & TOL & 0.881 & 0.73 \\
\hline & $(7.20-180)$ & MEK & 0.933 & 0.70 \\
\hline & $(1.1-1.47)$ & DEM & 1.240 & 0.67 \\
\hline & & $1-\mathrm{CB}$ & 0.967 & 0.71 \\
\hline & & CHL(62.0) & 7.40 & 0.50 \\
\hline \multirow[t]{6}{*}{ B } & $6: 0.50 \pm 0.10$ & THF(25.05) & 0.641 & 0.76 \\
\hline & $(3.16-14.4)$ & TOL & 1.318 & 0.69 \\
\hline & $(1.16-1.61)$ & MEK & 1.466 & 0.67 \\
\hline & & DEM & 1.897 & 0.64 \\
\hline & & $1-\mathrm{CB}$ & 4.446 & 0.55 \\
\hline & & CHL(81.0) & 6.30 & 0.50 \\
\hline
\end{tabular}

a Abbreviations, see text: Measurements were made at $30.0^{\circ} \mathrm{C}$, unless otherwise specified. dimensions may be estimated from good solvent data by employing an appropriate extrapolation procedure that separates the effects of the shortrange and long-range interactions. ${ }^{28-33}$ However, the extrapolation often results in an ambiguous estimate. $^{30,31}$ There is no advantage in using such methods so far as $\theta$-solvent data are available. The results of the anlysis are listed in Table V.

\section{Long-Range Interactions}

The interaction parameter $B$ was estimated by a viscosity equation recently proposed by Fujita et $a l .{ }^{34}$ for good solvent data.

$$
\begin{gathered}
{[\eta] M_{w}=1.05 K_{0}+0.83 \Phi_{0} B M_{w}{ }^{1 / 2}} \\
\left(\text { for } 1.2<\alpha_{\eta}{ }^{3}=[\eta] /[\eta]_{\theta}<2.3\right)
\end{gathered}
$$

The parameter $B$ is proportional to the binarycluster integral $\beta$ for a pair of segments within a chain consisting of $n$ such segments: ${ }^{3}$

$$
B=(4 \pi)^{-3 / 2} \beta\left(\frac{n}{M}\right)^{2}
$$

When the notations were brought into correspondence with the well-known Flory-Huggins notations based on the lattice theory, ${ }^{2-4}$ it was found that

$$
\beta=2\left(\frac{V_{s}}{N}\right)\left(\frac{1}{2}-\chi\right) ; n=\left(\frac{M \bar{v}}{V_{s}}\right)
$$

where $N$ was the Avogadro number; solvent molecules and segments were assumed to have the same volume $\left(V_{s} / N\right)$. Figures 3-6 show the plots of $[\eta] M_{w}{ }^{-1 / 2}$ vs. $M_{w}{ }^{1 / 2}$, from which the

Table V. Values of short-range interaction parameters for St-MMA model copolymers

\begin{tabular}{lcccccc}
\hline Code & $\begin{array}{c}\text { St-content } \\
m\end{array}$ & $\begin{array}{c}\text { Run number } \\
2 r\end{array}$ & $10^{4} K_{0}\left({ }^{\circ} \mathrm{C}\right)^{\mathrm{a}}$ & $10^{18}\left\langle s^{2}\right\rangle_{0} / M$ & $C_{\infty} \mathrm{b}$ & $\delta C_{\infty}$ \\
\hline PMMA & 0 & 0 & $5.0 \pm 0.5(30-85)$ & 5.44 & 6.95 & 0 \\
PSt & 0 & 0 & $8.0 \pm 0.5(<80)$ & 7.44 & 9.92 & 0 \\
& & & $6.8 \quad(81)$ & 6.69 & 8.61 & 0 \\
AL & $0.50 \pm 0.01$ & 1.00 & $8.0 \pm 0.2(61.0)$ & 7.44 & 9.73 & 1.30 \\
SM3 & $0.29 \pm 0.01$ & 0.51 & $6.6 \pm 0.2(68.0)$ & 6.55 & 8.45 & 0.64 \\
SMA & $0.52 \pm 0.01$ & 0.67 & $7.4 \pm 0.2(62.0)$ & 7.07 & 9.20 & 0.71 \\
SMA & 0.546 & 0.66 & $7.5^{\mathrm{c}}$ & 7.13 & 9.28 & 0.72 \\
SM5 & $0.56 \pm 0.01$ & 0.66 & $7.5 \pm 0.2(64.0)$ & 7.13 & 9.28 & 0.72 \\
SM7 & $0.70 \pm 0.01$ & 0.52 & $7.7 \pm 0.2(64.0)$ & 7.26 & 9.54 & 0.51 \\
B & $0.50 \pm 0.10$ & 0 & $6.3 \pm 0.4(81.0)$ & 6.35 & 7.86 & 0.12 \\
\hline
\end{tabular}

a From $[\eta]_{\Theta}$ data on $\mathrm{CHL}$ at the temperatures as indicated.

b For block copolymers, $10^{4} K_{0}=6.8$ for PSt was used (ref. 38); otherwise 8.0 for PSt was used.

c MEK data (ref. 4). 
Table VI. Values of long-range interaction parameters for St-MMA model copolymers in various solvents

\begin{tabular}{|c|c|c|c|c|}
\hline Code & $\bar{v}$ & $10^{30} B$ & $\chi$ & $\begin{array}{c}\chi_{\mathrm{c}^{\mathrm{a}^{\mathrm{lc}}}} \\
\text { (Eq. } 11 \mathrm{~b})\end{array}$ \\
\hline \multicolumn{5}{|c|}{$\operatorname{THF}\left(25.05^{\circ} \mathrm{C}\right) ; V_{s}=82.3$} \\
\hline $\mathrm{PSt}^{\mathrm{a}}$ & 0.9102 & 61.5 & 0.417 & \\
\hline PMMA $^{b}$ & 0.8085 & 42.1 & 0.429 & \\
\hline $\mathrm{AL}$ & 0.8604 & 60.9 & 0.409 & \\
\hline $\mathrm{SMA}^{\prime \prime b}$ & 0.861 & 89.4 & 0.357 & 0.413 \\
\hline B & 0.8604 & 52.4 & 0.421 & 0.423 \\
\hline \multicolumn{5}{|c|}{ TOL $\left(30.0^{\circ} \mathrm{C}\right) ; V_{s}=107.4$} \\
\hline $\mathrm{PSt}^{\mathrm{c}}$ & 0.917 & 62.5 & 0.390 & \\
\hline PMMA $^{d}$ & 0.807 & 23.8 & 0.446 & \\
\hline $\mathrm{AL}$ & 0.863 & 53.6 & 0.395 & \\
\hline SM3 & 0.840 & 34.6 & 0.427 & 0.418 \\
\hline SMA & 0.865 & 47.0 & 0.408 & 0.402 \\
\hline SMA $^{\prime e}$ & 0.868 & 48.0 & 0.406 & 0.401 \\
\hline SM5 & 0.870 & 47.7 & 0.407 & 0.400 \\
\hline SM7 & 0.885 & 58.4 & 0.389 & 0.395 \\
\hline B & 0.863 & 45.2 & 0.412 & 0.418 \\
\hline \multicolumn{5}{|c|}{$\operatorname{MEK}\left(30.0^{\circ} \mathrm{C}\right) ; V_{s}=90.8$} \\
\hline $\mathrm{PSt}^{\mathrm{f}}$ & 0.9078 & 10.7 & 0.484 & \\
\hline PMMAg & 0.7993 & 28.3 & 0.445 & \\
\hline $\mathrm{AL}$ & 0.8546 & 19.9 & 0.466 & \\
\hline SMA & 0.8568 & 22.3 & 0.462 & 0.466 \\
\hline SMA $^{\prime e}$ & 0.8570 & 27.6 & 0.447 & 0.467 \\
\hline B & 0.8546 & 25.6 & 0.457 & 0.464 \\
\hline \multicolumn{5}{|c|}{$\operatorname{DEM}\left(30.0^{\circ} \mathrm{C}\right) ; V_{s}=165.4$} \\
\hline $\mathrm{PSt}^{\mathrm{h}}$ & 0.929 & -6.5 & 0.518 & \\
\hline PMMA & 0.810 & 14.9 & 0.449 & \\
\hline $\mathrm{AL}$ & 0.866 & 13.7 & 0.458 & \\
\hline SM3 & 0.843 & 19.4 & 0.438 & 0.456 \\
\hline SMA & 0.868 & 18.5 & 0.445 & 0.468 \\
\hline SM5 & 0.873 & 16.4 & 0.451 & 0.471 \\
\hline SM7 & 0.888 & 14.9 & 0.457 & 0.484 \\
\hline B & 0.866 & 18.5 & 0.444 & 0.482 \\
\hline \multicolumn{5}{|c|}{$1-\mathrm{CB}\left(30.0^{\circ} \mathrm{C}\right) ; V_{s}=105.7$} \\
\hline PSt & 0.920 & 13.7 & 0.477 & \\
\hline PMMA $^{i}$ & 0.820 & -4.2 & 0.509 & \\
\hline $\mathrm{AL}$ & 0.871 & 26.8 & 0.448 & \\
\hline SM3 & 0.850 & 13.4 & 0.473 & 0.476 \\
\hline SMA & 0.873 & 25.9 & 0.451 & 0.462 \\
\hline SM5 & 0.877 & 23.8 & 0.455 & 0.461 \\
\hline SMG & 0.891 & 39.1 & 0.443 & 0.463 \\
\hline B & 0.871 & 8.0 & 0.485 & 0.493 \\
\hline \multicolumn{5}{|c|}{ CHL $\left(81.0^{\circ} \mathrm{C}\right) ; V_{s}=111.0(c f$. Table III $)$} \\
\hline PSt & 0.957 & 0.0 & 0.500 & \\
\hline PMMA & 0.840 & 1.0 & 0.498 & \\
\hline AL & 0.900 & 20.0 & 0.462 & \\
\hline SM3 & 0.875 & 9.52 & 0.481 & 0.479 \\
\hline SM5 & 0.907 & 12.8 & 0.476 & 0.474 \\
\hline SM7 & 0.923 & 14.3 & 0.474 & 0.480 \\
\hline B & 0.905 & 0.0 & 0.500 & 0.499 \\
\hline
\end{tabular}

G. V. Schulz and H. Bauman, Makromol. Chem., 114, 122 (1968).

b A. Dondos and H. Benoit, ibid., 118, 165 (1968).

c Ref. 33 .

d T. G Fox, Polymer, 3, 111 (1962).

e Ref. 4.

f P. Outer, C. I. Carr, and B. H. Zimm, J. Chem. Phys., 18, 830 (1950)

g S. N. Chinai, J. D. Matlack, A. L. Resnick, and R. J. Samuels, J. Polym., Sci., 17, 391 (1955).

h From $A_{2}$-data: T. A. Orofino, J. Chem. Phys., 45, 4310 (1966).

i From $A_{2}$-data; G. V. Schulz, A. Haug, and R. Kirste, Z. Physik. Chem. (NF), 38, 1 (1963).

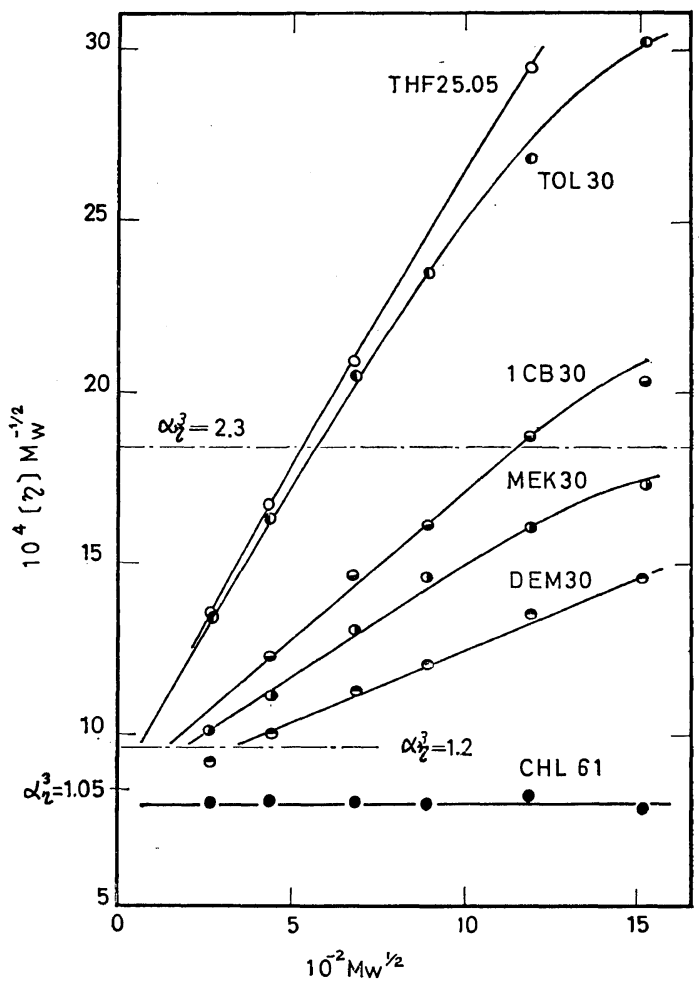

Figure 3. Plots of $[\eta] M_{w}^{-1 / 2} v s . M_{w}^{1 / 2}$ for St-MMA alternating copolymers in various solvents and temperature as indicated. See text for solvent codes.

long-range interaction parameters are estimated according to Eqs. $6 \mathrm{a}, \mathrm{b}$, and c. The results are summarized in Table VI.

\section{DISCUSSION}

\section{Short-Range Interactions}

Previously Stockmayer et al. ${ }^{4}$ proposed a simple relation for $\left\langle r^{2}\right\rangle_{0}$ of an $a-b$ binary copolymer as

$$
\frac{\left\langle r^{2}\right\rangle_{0}}{M}=x\left(\frac{\left\langle r^{2}\right\rangle_{0}}{M}\right)_{A}+(1-x)\left(\frac{\left\langle r^{2}\right\rangle_{0}}{M}\right)_{B}
$$

Here the quantities in parentheses are each characteristic of a single type of monomer, and hence, are deduced from those of the parent homopolymers. It may be easily shown that Eq. 7a is equivalent to the following relation of characteristic ratios:

$$
C_{\infty}=m C_{\infty}^{A}+(1-m) C_{\infty}{ }^{B}
$$

where the superscripts denote the quantities 


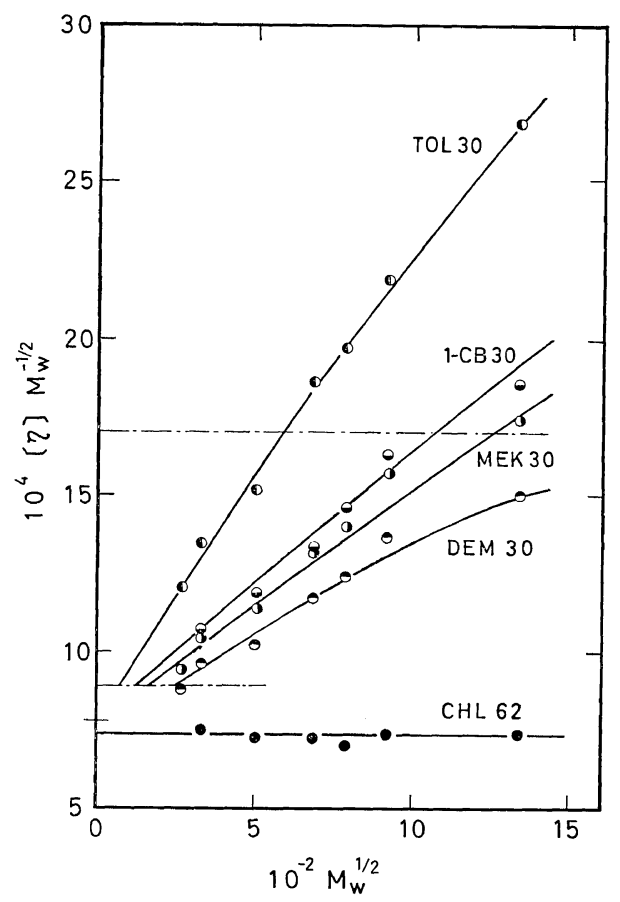

Figure 4. Plots of $[\eta] M_{w}^{-1 / 2}$ vs. $M_{w^{1 / 2}}$ for St-MMA statistical copolymers with the azeotropic composition in various solvents and temperature as indicated (cf. Figure 3).

characteristic of the parent homopolymers. Here we define an extra-interaction term $\delta C_{\infty}$ as

$$
\delta C_{\infty}=C_{\infty}-\left[m C_{\infty}{ }^{A}+(1-m) C_{\infty}{ }^{B}\right]
$$

The values are listed in the last column of Table V.

At first glance the $K_{0}$ values of the block copolymers, Eqs. $7 \mathrm{a}$ and $\mathrm{b}$ proposed by Stockmayer et $a .^{4}{ }^{4}$ appear to be satisfactory. However, close inspection reveals that Eq. $7 \mathrm{~b}$ gives somewhat larger values of $C_{\infty}$, if values of $10^{4} K_{0}=8.0$ for PSt and 5.0 for PMMA are employed. On the other hand, Reiss and Benoit ${ }^{38}$ suggest that the unperturbed dimensions of PSt show anomalous temperature dependence around 75 to $80^{\circ} \mathrm{C}$ : In fact, for the PSt-CHL system at $83.5^{\circ} \mathrm{C}$, the value $10^{4} K_{0}=6.7$ was reported. ${ }^{38}$ The decrease in $K_{0}$ of this system was confirmed also by our measurements at $81^{\circ} \mathrm{C}$. If this low value is to be used in Eqs. 5 and 7a for PSt, Eq. 7b yields a value of $C_{\infty}$ somewhat smaller than that determined by experiments ( $c f$. Table V). The discrepancy implies that the effects of long-range

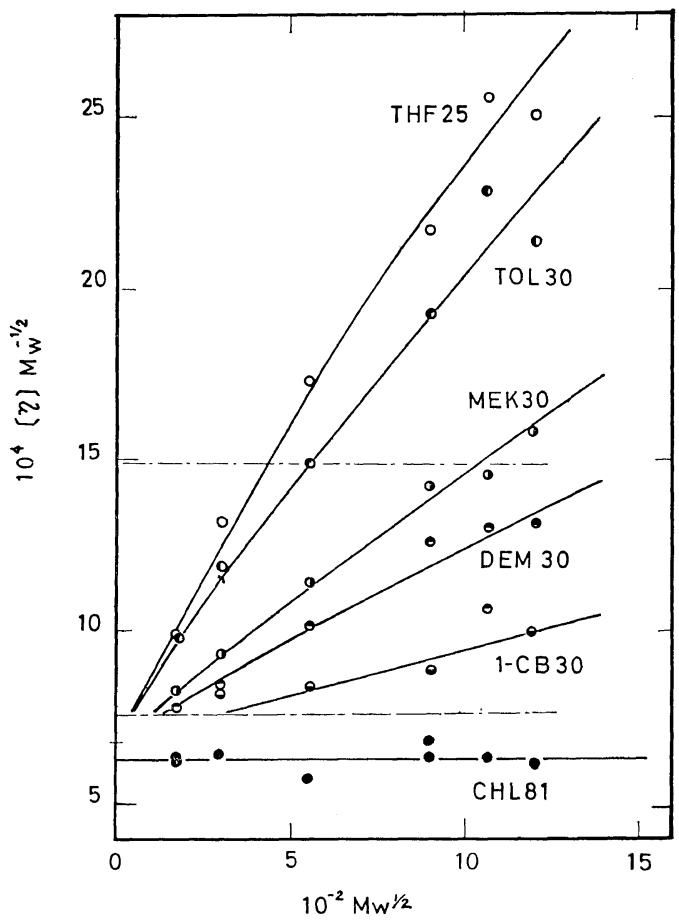

Figure 5. Plots of $[\eta] M_{w}^{-1 / 2}$ vs. $M_{w^{1 / 2}}$ for block copolymers of PMMA-PSt-PMMA type with nearly equimolar composition in various solvents and temperature as indicated (cf. Figure 3).

interactions might not be completely absent in this condition.

Turning our attention to the alternating and statistical copolymer data, we find that their characteristic ratios are distinctly larger than those expected from Eq. $7 \mathrm{~b}$ with the values $10^{4} K_{0}=8.0$ for PSt and 5.0 for PMMA (cf. Table VI). The extra-terms $\delta C_{\infty}$, as demonstrated in Figure 7, are found to be roughly proportional to the population of dyads of unlike monomers, i.e. to the run number $2 r=P_{a b}+P_{b a}$. This means that $C_{\infty}$ and $\delta C_{\infty}$ of a statistical copolymer with composition $m$ and run number $2 r$ may be approximated as

$$
\begin{aligned}
& C_{\infty}=(m-r) C_{\infty}{ }^{A}+(1-m-r) C_{\infty}{ }^{B}+2 r C_{\infty}{ }^{A B} \\
& \delta C_{\infty}=2 r \Delta=2 r\left[C_{\infty}{ }^{A B}-\frac{1}{2}\left(C_{\infty}{ }^{A}+C_{\infty}{ }^{B}\right)\right]
\end{aligned}
$$

where $C_{\infty}{ }^{A B}$ is the value of the alternating copolymer; $(m-r),(1-m-r)$, and $2 r$ are respectively 


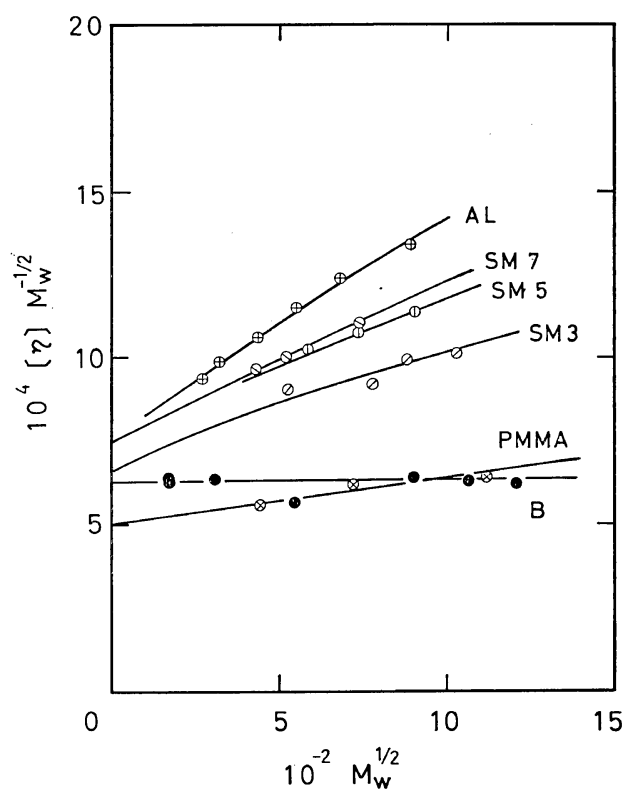

Figure 6. Plots of $[\eta] M_{w}^{-1 / 2} v s . M_{w}^{1 / 2}$ for St-MMA alternating and statistical copolymers, and the parent homopolymers in cyclohexanol at $81.0^{\circ} \mathrm{C}$. See text for sample codes.

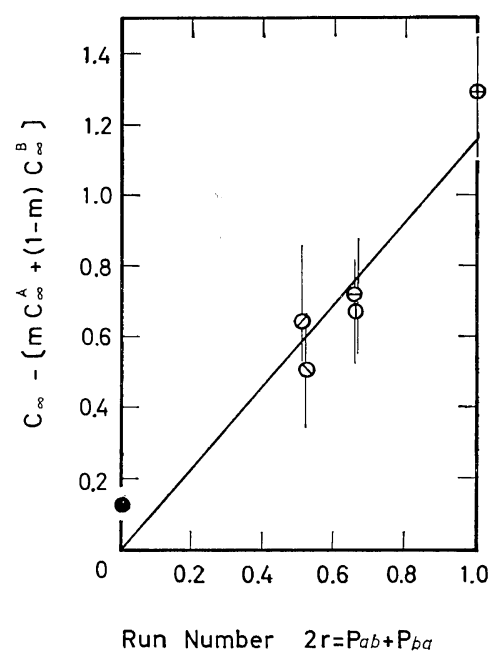

Figure 7. Plots of the extra-short-range interaction term $\delta C_{\infty} v s$. the run number $2 r$ for St-MMA model copolymers ( $c f$. Table V). See Figure 6 for symbols.

proportional to the population of $a a, b b$, and $(a b+b a)$ dyads. The relation is equivalent to those previously reported by Nomura and Iwachi$\mathrm{do}^{39}$ and also by Utracki and Simha. ${ }^{10}$ The value of $\Delta$ is positive and nonzero for the St-MMA alternating and statistical copolymers. In contrast to the above result, Matsuda, Yamano, and Inagaki ${ }^{40}$ report that for St-methyl acrylate (MA) statistical copolymers the extra-term is absent, i.e., $\Delta=0$.

Apparently the extra-term for a particular system should be related to both tactic and chemical placement of monomers along the copolymer chain. In this connection, Ito and Yamashita $^{24 b}$ have reported on the basis of high-resolution NMR spectra that the tactic placements of monomers in St-MA and St-MMA statistical copolymers are considerably different: The coisotacticity, which is defined as the probability of finding dyads of the unlike monomers in which phenyl and methyl ester groups have the same stereochemical placement, is 0.8 for St-MA copolymers, whereas it is 0.5 (atactic) for St-MMA copolymers. By judging from the three methoxy proton peaks at $6.6,7.0$ and $7.6 \tau$ found in the $\mathrm{nmr}$ spectrum of the alternating copolymer ( $c f$. Figure 1), it is also atactic. ${ }^{14}$ This might be a reason for the difference in $\Delta$ of the two systems. ${ }^{40}$ On the other hand, recent Monte Carlo calculations $^{10}$ have suggested that the effect of tactic placement on $C_{\infty}$ of assymmetric vinyl chains becomes significant only for predominantly stereoregular chains. If such is the case, a more likely reason for the difference in $\Delta$ of the two systems could be the difference in the nature of hindrance potentials rather than the difference in the tactic placement. Both PSt and PMA chains are asymmetric vinyl chains, and the preferred conformations of any given dyads would be essentially unaltered, when St-units in a PSt chain are replaced by MA-units or vice versa, i.e., when a copolymer chain is formed from $\mathrm{St}$ and MA monomers. ${ }^{37}$ On the contrary, alternate skeletal carbon atoms in PMMA chains bear two substituents which are dissimilar but both substantially bulky. Hence steric repulsions owing to them make the PMMA chain behave more like a symmetric chain. (Probably this is responsible for the rather small $C_{\infty}$ value of atactic and syndiotactic PMMA chains in comparison with PMA and PSt chains.) The replacement of St-units in a PSt chain by MMA units or vice versa necessarily disturbs the main chain conformations. 
This might be responsible for the nonzero $\Delta$ value of the St-MMA systems. ${ }^{37}$ In this connection, it would be interesting to note that our preliminary results $^{41}$ suggest $\Delta$ being nearly zero for $\alpha$-methyl styrene (MSt)-MMA systems. The coisotacticity for this system was reported to be 0.25 , in contrast to the St-MA system. ${ }^{24 b}$ Both MSt and MMA are $\alpha$-substituted vinyl monomers, and again the replacement of the monomers in either of the homopolymer chains might not disturb the main chain conformation.

\section{Long-Range Interactions}

The results listed in Table VI clearly indicate the long-range interaction parameters to be dependent on the sequential arrangement as well as on the composition. Again Stockmayer et $a l .{ }^{4}$ have suggested a simple relation for $\chi$ of a binary copolymer as

$$
\chi=m \chi_{a}+(1-m) \chi_{b}-m(1-m) \chi_{a b}
$$

where $\chi_{a}$ and $\chi_{b}$ are the parameters characterizing segment-solvent interactions for homopolymers $\mathrm{A}$ and $\mathrm{B}$, respectively (for brevity, subscript $s$ denoting solvent is omitted); and $\chi_{a b}$ is the paramer characterizing the $a-b$ interactions. Eq. $8 \mathrm{a}$ is equivalent to $\beta$ expressed as a quadratic function of composition:

$$
\begin{gathered}
\beta=m \beta_{a}+(1-m) \beta_{b}+2 m(1-m) \Delta \beta_{a b} \\
\Delta \beta_{a b}=\frac{V_{s}}{N} \chi_{a b}
\end{gathered}
$$

When the homopolymers A and B are incompatible, i.e., the $\chi_{a b}$ is positive, the parameter $\chi$ is smaller (or the $\beta$ is larger) so that the solvent is better toward the $a-b$ copolymers. In general, the parameter $\chi$ involves a contribution to the enthalpy and the entrophy of mixing characterizing nearest-neighbor interactions due to solute-solvent contacts. For copolymer systems both terms would be quadratic functions of composition. However, the contribution to the entropy is expected to be considerably smaller than that to the enthalpy so that the parameters may be approximated in terms of the Hildebrand-Scott solubility parameter ${ }^{42} \delta$ 's as

$$
\delta_{i j}=\left(\frac{V_{s}}{N}\right) \frac{\left(\delta_{i}-\delta_{i}\right)}{k T} \quad(i, j=a, b \text { or } s)
$$

As pointed out by Stockmayer, ${ }^{4}$ Eqs. 9a and b would be most nearly correct for such chains in which the average sequence lengths of either monomers are (i) much shorter than the total length of the copolymer chain, but are still (ii) sufflciently long so as to preserve the nature of the homopolymer segments. In a strict sense, the assumption (i) is invalid for block copolymers. ${ }^{77}$ In fact, a perturbation calculation of $\left\langle s^{2}\right\rangle$ up to the single contact term gives $\beta$ for an equimolar block copolymer of $\mathrm{AB}$ type $\mathrm{as}^{7}$

$$
\beta=0.50\left(\beta_{a}+\beta_{b}\right)+0.3064 \beta_{a b}
$$

and a similar calculation for an equimolar block copolymer of symmetric BAB type (i.e., both B's have the same length) $:^{37}$

$$
\beta=0.64 \beta_{a}+0.36 \beta_{b}+0.31 \Delta \beta_{a b}
$$

Nevertheless, Eqs. 9a and b would be semiquantitatively a good approximation for block copolymers. ${ }^{9}$

On the contrary, the assumption (ii) would be invalid for alternating and statistical copolymers. As is obvious from Table VI, the difference between the values of $B$ of these two copolymers indicates that the effects of the sequential arrangement are not negligible. To incorporate the effects explicitly into $\chi$, we arbitrarily consider a dyad as a segment. ${ }^{11}$ In other words, we consider the influence of one neighboring monomer unit for the interaction between a given monomer and a solvent. Then we assume a function for $\chi$ as

$$
\chi=\sum p_{i} \chi_{i}-\sum_{i<j} \sum_{i} p_{i} p_{j} \chi_{i j}
$$

where we tentatively assign dyads $a a, b b$, and $a b(=b a)$ as segments 1,2 , and 3 , respectively; and $p_{i}$ 's are the fractions of such dyads. Of course, one may assume a longer sequence (e.g., a triad, tetrad etc.) as a segment, and represent $\chi$ by introducing a larger number of parameters, and still preserving the form of Eq. 11. However, such extension is meaningless at the moment, since the segment volume, in the first place, is assumed to be comparable to the solvent volume, and secondly a multiparameter equation itself is useless, unless each parameter can be determined separately. The Eq. 11a with the dyad approximation involves six different parameters: Three $\chi_{i}$ 's are determined from the parent homo- 
polymer and the alternating copolymer data, while each of three $\chi_{i j}$ 's could be, in principle, identified with an interaction parameter which characterizes the $i-j$ interaction in the ternary system solventpolymer $i$-polymer $j$. For the time being, to avoid the difficulty of estimating $\chi_{i j}$ 's, we further assume Eq. 10 to be valid also for the nearestneighbor interactions involving the segments (dyads) such as defined here. Then we obtain $\chi$ for a copolymer with composition $m$ and run number $2 r$ as

$$
\chi^{1 / 2}=(m-r) \chi_{1}{ }^{1 / 2}+(1-m-r) \chi_{2}{ }^{1 / 2}+2 r \chi_{3}{ }^{1 / 2}
$$

where $\chi_{1}, \chi_{2}$, and $\chi_{3}$ may be identified respectively with the interaction parameters of the homopolymers A, B and the alternating copolymer with the pure solvent. ${ }^{37}$ The parameter $\chi$ of a block copolymer with composition $m$ would be approximately given by Eq. $11 \mathrm{~b}$ simply by setting $r=0$. This simple equation explains, at least qualitatively, the composition dependence of the $\theta$ temperatures of the statistical and block copolymer-CHL systems which is indicated in Table II and also was reported previously. ${ }^{8,13,18}$

In the last two columns of Table VI the values of $\chi$ for the statistical and block copolymers are compared with those calculated by Eq. 11b. Figure 8 shows the plots of $\chi v s . m$ for the THF, TOL, MEK, and CHL $\left(81.0^{\circ} \mathrm{C}\right)$ systems. Although quantitative agreement between the experimental and calculated values is not quite satisfactory, the basic concept involved in Eq. 11a will be substantially correct. The segmentsolvent interactions are affected by the local structure (perhaps over a few skeletal bonds) of copolymer chains, and obviously they cannot be described by those of the monomeric units alone. The situation must also be true for homopolymer systems: for example, the same concept would help to describe the dependence of their longrange interactions on the stereoregularity of homopolymer chains. The poor agreement between the experimental and calculated values of $\chi$ for the St-MMA model copolymers appears to be due partly to (i) an approximation involved in Eq. 10 (by which Eq. 11a could be reduced to Eq. 11b), and partly to (ii) a specific definition of a segment, for which we have tentatively assumed

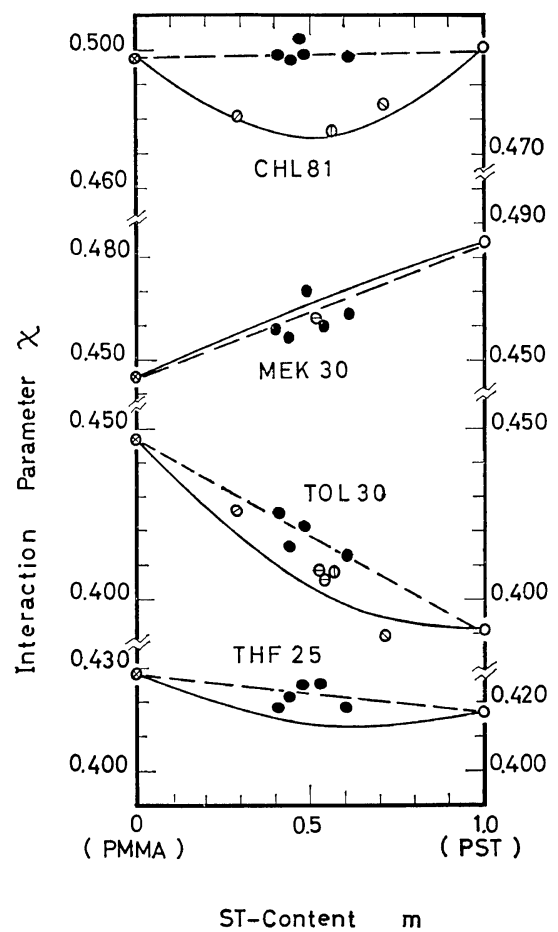

Figure 8. Plots of interaction parameter $\chi$ vs. composition $m$ for St-MMA statistical and block copolymers. See Figure 6 for symbols. Solid curves are those calculated by Eq. $11 \mathrm{~b}$ for the statistical copolymers; and dashed curves for the block copolymers.

each dyad as a segment. The latter reason might be important for the DEM system, since the DEM molecule has a somewhat complex structure and about 1.6 times larger volume in comparison with other solvents and the monomers. One more remark should be added here for behavior of the block copolymers in DEM and 1-CB, in which either one of the constituent blocks would become unstable. Particularly in 1-CB, two PMMA-subblocks in a chain would possibly undergo the intramolecular aggregation. Some evidence suggests this should be the case. ${ }^{13 a}$ This may also be responsible for the poor agreement between the experimental and calculated values of $\chi$ for the block copolymers in these solvents.

\section{CONCLUSION}

As a result of the analysis of dilute solution 
characteristics of the St-MMA model copolymers, i.e., the alternating, statistical and block copolymers of PMMA-PSt-PMMA type all with nearly equimolar composition, it was found that both the short-range and long-range interactions are dependent on the sequential arrangement as well as on the composition of monomers.

The short-range interactions of the block copolymers, as expressed by the characteristic ratios, appear to be approximated by a composition average of those of the parent homopolymers, as suggested previously by Stockmayer. ${ }^{4}$ The slight discrepancy between the composition average and the experimental value obtained in CHL at $81.0^{\circ} \mathrm{C}$ implies that the effects of long-range interactions on the block copolymer chains might not be completely absent at this condition. On the other hand, the characteristic ratios of the alternating and statistical copolymers are found to be larger than the composition average of those of the parent homopolymers: the deviation, the extra short-range interaction term, is very roughly proportional to the population of the unlike-monomer dyads, and hence, is the largest in the alternating copolymer. This result is in contrast with those of St-MA and MSt-MMA statistical copolymers, in either of which the extra-term is negligible. The extra-term found in the St-MMA systems may be indicative of particular relationships among the hindrance potentials of an $\alpha$-substituted vinyl chain (PMMA), an asymmetric vinyl chain (PSt), and a copolymer chain consisting of these two different types of monomers.

The long-range interactions in the model copolymer systems are interpreted by assuming dyads as copolymer segments. Thus, the interaction parameters $\chi$ of the statistical and block copolymers may be expressed as a function of the composition and the run number in terms of the parameters characterizing the parent homopolymers and the alternating copolymer. The copolymer segment-solvent interactions cannot be described by those of the monomeric units alone, and the difference in the local structure (perhaps over a few consecutive bonds) along the chain should be taken into account.

Acknowledgement. The authors wish to thank Messers H. Suzuki and N. Donkai for their as- sistance in carrying out experiments. H. O. wishes to express his thanks to the Japan Society for the Promotion of Science, and Y. M. to Teijin and Co. for the fellowship grants. A financial support from the Ministry of Education is gratefully acknowledged.

\section{REFERENCES}

1. (a) M. V. Volkenstein, Configurational Statistics of Polymeric Chains, translated by S. N. Timasheff and H. J. Timasheff, (Interscience Publishers, New York, 1963); (b) T. M. Birshtein and O. B. Ptitsyn, Conformations of Macromolecules, translated by S. N. Timasheff and M. J. Timasheff, (Interscience Publishers, New York, 1966); (c) P. J. Flory, Statistical Mechanics of Chain Molecules, (Interscience Publishers, New York, 1969).

2. P. J. Flory, Principles of Polymer Chemistry, (Cornell University Press, Ithaca, New York, 1953).

3. W. H. Stockmayer, Makromol. Chem., 35, 54 (1960).

4. W. H. Stockmayer, L. D. Moore, Jr., M. Fixman, and B. N. Epstein, J. Polym. Sci., 16, 517 (1955).

5. For a review, see: H. Benoit, Ber. Bunsenges. Physik. Chem., 70, 286 (1966).

6. H. Utiyama, Dissertation, Kyoto University, 1963.

7. D. Froelich and H. Benoit, Makromol. Chem., 92, 224 (1966).

8. T. Kotaka, Y. Murakami, and H. Inagaki, $J$. Phys. Chem., 72, 829 (1968).

9. L. A. Utracki, R. Simha, and L. J. Fetters, $J$. Polym. Sci., Part A2, 6, 2051 (1968).

10. L. A. Utracki and R. Simha, Macromolecules, 1, 505 (1968).

11. Y. Murakami, Dissertation, Kyoto University, 1968.

12. T. Kotaka, H. Ohnuma, and H. Inagaki, Bull. Inst. Chem. Res., Kyoto Univ., 46, 107 (1968).

13. (a) H. Ohnuma, Dissertation, Kyoto University, 1969; (c) H. Ohnuma, T. Kotaka, and H. Inagaki, Polymer, 10, 501 (1969); (c) T. Kotaka, H. Ohnuma, and H. Inagaki, ibid., 10, 517 (1969).

14. (a) M. Hirooka, Kobunshi, (High Polymer, Japan) 16, 1172 (1967); (b) M. Hirooka, H. Yabuuchi, J. Iseki, and Y. Nakai, J. Polym. Sci., Part A1 6, 1381 (1968).

15. (a) G.E.Ham, Ed. Copolymerization (Interscience Publishers, New York, 1964); (b) F. R. Mayo, Ber. Bunsenges. Physik. Chem., 70, 233 (1966); 
(c) F. R. Mayo and F. M. Lewis, J. Amer. Chem. Soc., 66, 1594 (1954).

16. (a) M. Szwarc and A. Rembaum, J. Polym. Sci., 22, 189 (1956); (b) M. Szwarc, Adv. Polym. Sci., 2, 275 (1960); (c) J. R. Urwin and J. M. Stearne, Makromol. Chem., 78, 194, 204 (1964).

17. D. Freyss, P. Rempp and H. Benoit, J. Polym. Sci., Part B2, 217 (1964).

18. T. Kotaka, H. Ohnuma, and Y. Murakami, J. Phys. Chem., 70, 4099 (1966).

19. (a) P. J. Flory, J. Chem. Phys., 10, 51 (1942); (b) A. R. Shultz and P. J. Flory, J. Amer. Chem. Soc., 74, 4760 (1952); 75, 3888 (1953).

20. B. H. Zimm, J. Chem. Phys., 16, 1093, 1099 (1948).

21. (a) W. Bushuk and H. Benoit, Compt. Rend., 24, 3169 (1958); Can. J. Chem., 36, 1616 (1958): (b) H. Benoit and C. Wippler, J. Chim. Phys., 57, 524 (1960): (c) M. Leng and H. Benoit, ibid., 58, 480 (1960); J. Polym. Sci., 57, 263 (1962).

22. W. H. Stockmayer, J. Chem. Phys., 13, 199 (1945).

23. (a) H. J. Harwood and W. M. Ritchey, J. Polym. Sci., Part B 2, 601 (1964): (b) H. J. Harwood, Angew. Chem., 77, 405 (1965).

24. (a) F. A. Bovey, J. Polym. Sci., 62, 197 (1962):

(b) K. Ito, S. Iwase, K. Umehara, and Y. Yamashita, J. Macromol. Sci., Part A, 1, 891 (1967); Kogyo Kagaku Zasshi, 70, 993 (1967).

25. H. Inagaki, H. Matsuda, and F. Kamiyama, Macromolecules, 1, 520 (1968).

26. (a) T. G Fox and P. J. Flory, J. Polym. Sci., 14, 315 (1954): (b) R. B. Beevers and E. T. White, Trans. Faraday Soc., 56, 744 (1960).

27. (a) G. V. Schulz, A. Haug, and R. Kirste, $Z$. Physik. Chem. (NF) 38, 1 (1963): (b) T. A. Orofino, J. Chem. Phys., 45, 4310 (1966).
28. M. Kurata and W. H. Stockmayer, Adv. Polym. Sci., 3, 196 (1963).

29. W. H. Stockmayer and M. Fixman, J. Polym. Sci., Part C, 1, 137 (1963).

30. H. Inagaki, H. Suzuki, and M. Kurata, ibid., Part C, 15, 409 (1966).

31. J. M. G. Cowie, Polymer, 7, 487 (1966).

32. P. J. Flory, Makromol. Chem., 98, 128 (1966).

33. G. C. Berry, J. Chem. Phys., 44, 4550 (1966); 46, 1338 (1967).

34. (a) T. Norisuye, K. Kawahara, A. Teramoto, and H. Fujita, J. Chem. Phys., 49, 4330 (1968): (b) K. Kawahara, T. Norisuye, and H. Fujita, ibid., 49, 4339 (1968).

35. C. W. Pyun and M. Fixman, ibid., 42, 3838 (1965).

36. Th. G. Scholte, J. Polym. Sci., Part A2 5, 461 (1967).

37. T. Tanaka and T. Kotaka, to be published.

38. (a) C. Reiss and H. Benoit, Comp. Rend., 253, 268 (1961): (b) C. Reiss and H. Renoit, $J$. Polym. Sci., Part C 16 (6), 3079 (1968).

39. H. Nomura and T. Iwachido, IUPAC symposium on Macromolecular Chemistry, Tokyo-Kyoto, 1966, Preprint VI-52.

40. H. Matsuda, K. Yamano, and H. Inagaki, $J$. Polym. Sci., Part A2, 7, 609 (1969).

41. M. Nakatani, T. Tanaka, and T. Kotaka, to be Published.

42. (a) J. H. Hildebrand and R. L. Scott, The Solubility of Nonelectrolytes, (Rheinhold, New York, 1950): (b) R. L. Scott, J. Polym. Sci., 9, 423 (1952): (c) J. H. Hildebrand and R. L. Scott, Regular Solutions, (Prentice Hall, Englewood Cliffs, N. J., 1962). 